\title{
Triterpenoid Saponins from Leaves of Hedera pastuchowii
}

\author{
Vakhtang Mshvildadze,${ }^{a}$ Riad Elias, ${ }^{*}, b$ Robert Faure, ${ }^{c}$ David Rondeau, ${ }^{d}$ Laurent Debrauwer, ${ }^{e}$ \\ Genri Dekanosidze, ${ }^{a}$ Ether Kemertelidze, ${ }^{a}$ and Guy Balansard ${ }^{b}$ \\ ${ }^{a}$ Institute of Pharmacochemistry, Academy of Sciences of Georgia; 36, St. P. Sarajishvili, 380059 Tbilisi, Georgia: \\ ${ }^{b}$ Laboratoire de Pharmacognosie, Faculté de Pharmacie, Université de la Méditerranée; 27 Boulevard Jean Moulin, \\ 13385 Marseille, cedex 5, France: ${ }^{c}$ UMR 6009, Université d'Aix-Marseille III, Avenue Escadrille Normandie Niemen; \\ 13397 Marseille, cedex 20, France: ' Service Commun d'Analyses Spectroscopiques, Université d'Angers; 2 Bd Lavoisier, \\ 49045 Angers cedex, France: and ${ }^{e}$ INRA Centre de Recherche de Toulouse, Laboratoire des Xénobiotiques; 180, chemin \\ de Tournefeuille, BP 3, 31931 Toulouse, France. $\quad$ Received April 5, 2004; accepted July 29, 2004
}

Five new triterpenoid saponins, pastuchoside A (1), B (3), C (5), D (7) and E (9), were isolated from the leaves of Hedera pastuchowii. They have oleanolic acid or hederagenin as aglycone. The structures were established by NMR spectroscopy including gs (gradient selected)-COSY, gs-HSQC, gs-HSQC-TOCSY and gs-HMBC experiments, and mass spectrometry ( ESI-HR-MS). Heptaoside saponins, compounds 1 and 3, are described for the first time in the genus Hedera.

Key words Hedera pastuchowii; Araliaceae; triterpenoid saponin; NMR; MS; pastuchoside

In the course of our phytochemical investigation of Hedera genus growing in Georgia, ${ }^{1)}$ we have previously reported the isolation of triterpene saponins from leaves and berries of Hedera colchica ${ }^{2,3)}$ and their biological activities in vitro. $\alpha$-Hederin and hederacolchiside A were the more active against Candida glabra $\left(\mathrm{LD}_{100}=6.25 \mu \mathrm{g} / \mathrm{ml}\right)$ and dermatophytes sp. $\left.\left(\mathrm{LD}_{100}=12.5 \mu \mathrm{g} / \mathrm{ml}\right) .{ }^{4}\right)$ The antileishmanial activity of $\alpha$-hederin, $\beta$-hederin and hederacolchiside $\mathrm{A}_{1}$ in association with Pentamidine and Amphotericin B showed that subtoxic concentrations of these saponins enhance the efficiency of Pentamidine and Amphotericin B on the promastigote and the amastigote forms of the parasites. ${ }^{5,6}$

Hederacolchiside $A_{1}$ was strongly cytotoxic against malignant melanoma $\mathrm{M}_{4}$ Beu $\left(\mathrm{IC}_{50}=5 \mu \mathrm{M}\right){ }^{7)}$ This saponin exhibits a preferential cytotoxicity on pigmented melanoma cells and interacts specifically with melanin. ${ }^{8)}$ Hedera helix saponins were found to have acute and chronic anti-inflammatory effects in rats. ${ }^{9)}$

In this paper we describe the isolation and structure determination of five new triterpene saponins, named pastuchosides A (1), B (3), C (5), D (7) and E (9), from the microwave dried leaves of $H$. pastuchowii (Fig. 1). Their structures were established on the basis of various 2D-NMR experiments (COSY, HSQC, HSQC-TOCSY and HMBC). Saponins with seven sugars, compounds $\mathbf{1}$ and $\mathbf{3}$, are reported for the first time in the genus Hedera. In addition, four known saponins, hederacolchiside F (2), ${ }^{10)}$ hederacolchiside E (4), ${ }^{11)}$ hederasaponin $\mathrm{C}(\mathbf{6})^{12)}$ and hederasaponin B (8), ${ }^{13)}$ were isolated from this plant.

Pastuchoside B (3) was assigned the molecular formula $\mathrm{C}_{71} \mathrm{H}_{116} \mathrm{O}_{34}$ by electrospray ionisation high-resolution mass spectrometry (ESI-HR-MS) $\left([\mathrm{M}+\mathrm{Na}]^{+}\right.$quasi-molecular ion at $m / z$ 1535.7280; $\mathrm{C}_{71} \mathrm{H}_{116} \mathrm{NaO}_{34}$ requires $\mathrm{m} / z$ 1535.7246). Acid hydrolysis of $\mathbf{3}$ yielded arabinose, glucose and rhamnose as sugars identified by TLC and oleanolic acid as a genin moiety. The ${ }^{13} \mathrm{C}-\mathrm{NMR}$ spectrum exhibited seven anomeric carbons located at $\delta 105.7,105.2,104.3,102.9$, $102.9,102.0$ and 95.8 (Table 1). The resonances of C-3 at $\delta 90.6$ and C-28 at $\delta 178.1$ for pastuchoside B (3) were characteristic of a bisdesmoside. Interglycosidic and sugar-agly- cone linkages as well as signal and structural assignments of the sugars were deduced on the basis of the following arguments. First of all, the gs (gradient selected)-HSQC ${ }^{14)}$ spectral analysis displayed the connectivities network for the anomeric atoms. Then, a gs-HSQC-TOCSY ${ }^{15)}$ experiment showed for each sugar residue the intra-correlated peaks between anomeric proton and sugar carbons (Table 1). To illustrate this strategy, only four carbon signals at $\delta 79.1(\mathrm{CH})$, $77.0(\mathrm{CH}), 73.4(\mathrm{CH})$ and $64.1\left(\mathrm{CH}_{2}\right)$ showed correlations with the anomeric proton at $\delta 4.49(J=6.0 \mathrm{~Hz})$. The above evidence (number and nature of carbons, chemical shifts and coupling constants) indicated that this sugar was an $\alpha$-Larabinopyranosyl residue. Subsequent examination of the HSQC-TOCSY diagram also indicated the occurrence of three glucose and three rhamnose units (Table 1). It should be noted that the HSQC-TOCSY sequence is more helpful than normal TOCSY for structural elucidation of numerous sugar chains, since severe crowding of sugar resonances occurs in the ${ }^{1} \mathrm{H}-\mathrm{NMR}$ spectrum with increasing monosaccha-

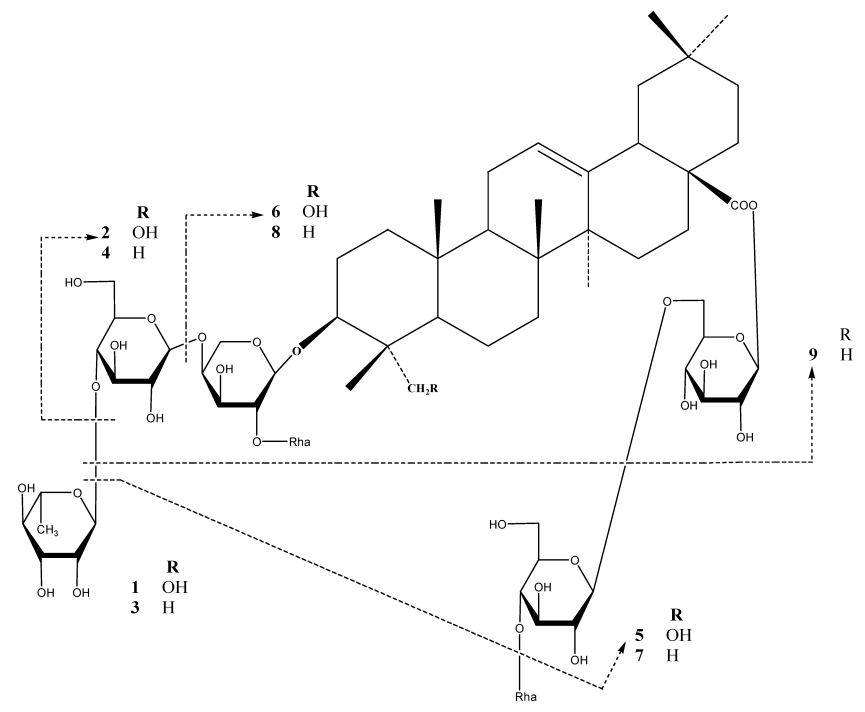

Fig. 1. Triterpenoid Glycosides Isolated from the Leaves of Hedera pastuchowii 
ride groups. The sequence and linkage site of the sugar moieties were established using long-range correlation peaks observed in the gs-HMBC ${ }^{16}$ ) diagram (Fig. 2). The HMBC spectrum showed correlations between C-3 ( $\delta$ 90.6) of oleanolic acid and $\mathrm{H}-1(\delta 4.49)$ of the arabinose, between C$2(\delta 77.1)$ and C-4 $(\delta$ 79.1) of the arabinose and H-1 $(\delta$ $5.20)$ of the first rhamnose and H-1 $(\delta 4.48)$ of the first glucose, respectively, and between C-4 ( $\delta 79.3)$ of the first glucose and H-1 $(\delta 4.87)$ of the second rhamnose. Similarly, the sequence of the trisaccharide chain at $\mathrm{C}-28$ was indicated by the cross peaks between C-6 ( $\delta$ 69.4) of the second glucose and $\mathrm{H}-1(\delta$ 4.42) of the third glucose, C-4 $(\delta 79.5)$ of the third glucose and H-1 $(\delta 4.86)$ of the third rhamnose. A cross peak between H-1 ( $\delta 5.38)$ of the second glucose and the ${ }^{13} \mathrm{C}$ resonance of the aglycone carboxy group ( $\delta$ 178.1) provided definitive evidence for an ester linkage between this trisaccharide chain and the genin. The complementary data from the gs-COSY spectrum was used to obtain a full assignment of the proton resonances (Table 2). Finally, the anomeric configuration for individual monosaccharides

Table 1. Anomeric Proton Connectivities from gs-HSQC and gs-HSQCTOCSY for Pastuchoside B (3) $\left(\delta\right.$ in ppm, $\left.\mathrm{CD}_{3} \mathrm{OD}\right)$

\begin{tabular}{crc}
\hline \hline & \multicolumn{2}{c}{${ }^{13} \mathrm{C}$} \\
\cline { 2 - 3 }${ }^{1} \mathrm{H}$ & \multicolumn{1}{c}{ HSQC } & HSQC-TOCSY \\
\hline $5.38, \mathrm{~d}, J=8.1 \mathrm{~Hz}$ & 95.8 & $78.2 ; 78.1 ; 73.8 ; 70.9 ; 69.4$ \\
$5.20, \mathrm{~d}, J=1.2 \mathrm{~Hz}$ & 102.0 & $73.9 ; 72.1 ; 72.1 ; 70.2 ; 18.0$ \\
$4.87, \mathrm{~d}, J=1.5 \mathrm{~Hz}$ & 102.9 & $73.7 ; 72.4 ; 72.2 ; 70.6 ; 17.9$ \\
$4.86, \mathrm{~d}, J=1.5 \mathrm{~Hz}$ & 102.9 & $73.7 ; 72.4 ; 72.2 ; 70.6 ; 17.9$ \\
$4.49, \mathrm{~d}, J=6.0 \mathrm{~Hz}$ & 105.2 & $79.1 ; 77.0 ; 73.4 ; 64.1$ \\
$4.48, \mathrm{~d}, J=7.8 \mathrm{~Hz}$ & 105.7 & $79.3 ; 76.9 ; 76.5 ; 75.4 ; 61.9$ \\
$4.42, \mathrm{~d}, J=7.5 \mathrm{~Hz}$ & 104.3 & $79.5 ; 76.8 ; 76.7 ; 75.2 ; 61.9$ \\
\hline
\end{tabular}

was deduced from the magnitude of the coupling constants of anomeric protons and ${ }^{13} \mathrm{C}-\mathrm{NMR}$ data. Based upon the above observations, the structure of $\mathbf{3}$ was elucidated as $3 \beta$ - $O$ - $\{\alpha$-L-rhamnopyranosyl- $(1 \rightarrow 2)$ - $[\alpha$-L-rhamnopyranosyl$(1 \rightarrow 4)$ - $\beta$-D-glucopyranosyl- $(1 \rightarrow 4)]-\alpha$-L-arabinopyranosyl $\}$ $28-O$ - $[\alpha$-L-rhamnopyranosyl-( $1 \rightarrow 4)-\beta$-D-glucopyranosyl$(1 \rightarrow 6)$ - $\beta$-D-glucopyranosyl]-oleanolate.

The molecular formula of pastuchoside A (1) was de duced to be $\mathrm{C}_{71} \mathrm{H}_{116} \mathrm{O}_{35}$ from ESI-HR-MS (measured mass: 1551.7191; theoretical mass for $\mathrm{C}_{71} \mathrm{H}_{116} \mathrm{NaO}_{35}$ : 1551.7195). TLC analysis of acid hydrolysis yielded glucose, rhamnose and arabinose as sugars and hederagenin as an aglycone. The ${ }^{13} \mathrm{C}$-NMR spectrum of the sugar moiety (Table 3 ) were similar to the data previously determined for pastuchoside B. Sugar arrangement was, consequently, the same for both
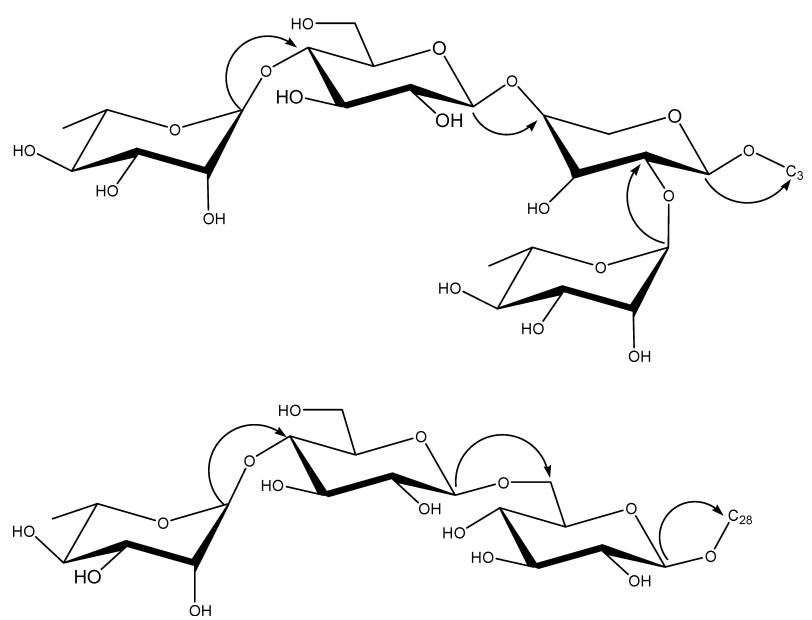

Fig. 2. Selected gs-HMBC Correlations $(\mathrm{H} \rightarrow \mathrm{C})$ Showing Interglycosidic Sugar Linkage for Pastuchoside B (3)

Table 2. ${ }^{1} \mathrm{H}-\mathrm{NMR}$ Data for Sugar Moieties of Saponins $\mathbf{1}$ and $\mathbf{3}\left(\mathrm{CD}_{3} \mathrm{OD}\right)$

\begin{tabular}{|c|c|c|c|c|c|c|c|}
\hline \multicolumn{2}{|c|}{ Sugar on C-3 $3^{a)}$} & \multirow{2}{*}{$\begin{array}{c}\mathbf{1} \\
4.49\end{array}$} & \multirow{2}{*}{$\begin{array}{c}\text { Sugar on C-28 } \\
4.47\end{array}$} & \multicolumn{2}{|l|}{3} & \multirow{2}{*}{$\begin{array}{c}\mathbf{1} \\
5.38\end{array}$} & \multirow{2}{*}{\begin{tabular}{c|}
$\mathbf{3}$ \\
5.38
\end{tabular}} \\
\hline ara & 1 & & & glc & 1 & & \\
\hline & 2 & 3.78 & 3.77 & & 2 & 3.35 & 3.36 \\
\hline & 3 & 3.83 & 3.83 & & 3 & 3.43 & 3.42 \\
\hline & 4 & 3.91 & 3.93 & & 4 & 3.41 & 3.41 \\
\hline & 5 & $4.14 ; 3.58$ & $4.15 ; 3.57$ & & 5 & 3.55 & 3.55 \\
\hline & & & & & 6 & $4.11 ; 3.82$ & $4.12 ; 3.83$ \\
\hline \multirow[t]{7}{*}{ rham } & 1 & 5.20 & 5.19 & & & & \\
\hline & 2 & 3.93 & 3.94 & glc & 1 & 4.42 & 4.43 \\
\hline & 3 & 3.72 & 3.71 & & 2 & 3.26 & 3.25 \\
\hline & 4 & 3.40 & 3.40 & & 3 & 3.48 & 3.48 \\
\hline & 5 & 3.89 & 3.89 & & 4 & 3.55 & 3.56 \\
\hline & 6 & 1.20 & 1.22 & & 5 & 3.38 & 3.38 \\
\hline & & & & & 6 & $3.85 ; 3.67$ & $3.85 ; 3.66$ \\
\hline \multirow[t]{7}{*}{ glc } & 1 & 4.48 & 4.46 & & & & \\
\hline & 2 & 3.34 & 3.33 & rham & 1 & 4.86 & 4.84 \\
\hline & 3 & 3.50 & 3.48 & & 2 & 3.66 & 3.67 \\
\hline & 4 & 3.59 & 3.59 & & 3 & 3.85 & 3.85 \\
\hline & 5 & 3.32 & 3.34 & & 4 & 3.41 & 3.41 \\
\hline & 6 & $3.85 ; 3.67$ & $3.86 ; 3.66$ & & 5 & 4.00 & 4.02 \\
\hline & & & & & 6 & 1.21 & 1.20 \\
\hline \multirow[t]{6}{*}{ rham } & 1 & 4.87 & 4.87 & & & & \\
\hline & 2 & 3.64 & 3.64 & & & & \\
\hline & 3 & 3.85 & 3.86 & & & & \\
\hline & 4 & 3.41 & 3.40 & & & & \\
\hline & 5 & 3.98 & 3.99 & & & & \\
\hline & 6 & 1.21 & 1.21 & & & & \\
\hline
\end{tabular}


Table 3. ${ }^{13} \mathrm{C}-\mathrm{NMR}$ Data for Sugar Moieties of Saponins 1, 3, 5, 7 and $9\left(\mathrm{CD}_{3} \mathrm{OD}\right)$

\begin{tabular}{|c|c|c|c|c|c|c|c|c|c|c|c|c|c|c|}
\hline & \multicolumn{3}{|c|}{1} & \multicolumn{2}{|c|}{3} & \multicolumn{3}{|c|}{5} & \multicolumn{3}{|c|}{7} & \multicolumn{3}{|c|}{9} \\
\hline \multicolumn{15}{|c|}{ Sugar on $C-3^{a)}$} \\
\hline \multirow[t]{5}{*}{ ara } & 1 & 105.2 & ara & 1 & 105.2 & ara & 1 & 105.2 & ara & 1 & 105.2 & ara & 1 & 105.2 \\
\hline & 2 & 77.1 & & 2 & 77.0 & & 2 & 76.9 & & 2 & 77.1 & & 2 & 77.0 \\
\hline & 3 & 73.4 & & 3 & 73.4 & & 3 & 73.4 & & 3 & 73.3 & & 3 & 73.4 \\
\hline & 4 & 79.1 & & 4 & 79.1 & & 4 & $77.8^{b)}$ & & 4 & $77.8^{b)}$ & & 4 & $77.9^{b)}$ \\
\hline & 5 & 64.2 & & 5 & 64.1 & & 5 & 64.2 & & 5 & 64.2 & & 5 & 64.1 \\
\hline \multirow[t]{6}{*}{ rham } & 1 & 102.0 & rham & 1 & 102.0 & rham & 1 & 101.9 & rham & 1 & 101.9 & rham & 1 & 102.0 \\
\hline & 2 & 72.1 & & 2 & 72.1 & & 2 & 72.1 & & 2 & 72.1 & & 2 & 72.1 \\
\hline & 3 & 72.1 & & 3 & 72.1 & & 3 & 72.1 & & 3 & 72.1 & & 3 & 72.1 \\
\hline & 4 & 73.9 & & 4 & 73.9 & & 4 & 73.9 & & 4 & 73.9 & & 4 & 73.9 \\
\hline & 5 & 70.2 & & 5 & 70.2 & & 5 & 70.1 & & 5 & 70.2 & & 5 & 70.2 \\
\hline & 6 & 18.0 & & 6 & 18.0 & & 6 & 17.9 & & 6 & 17.9 & & 6 & 17.9 \\
\hline \multirow[t]{6}{*}{ glc } & 1 & 105.7 & glc & 1 & 105.7 & glc & 1 & 106.1 & glc & 1 & 105.9 & glc & 1 & 106.0 \\
\hline & 2 & 75.4 & & 2 & 75.4 & & 2 & 75.3 & & 2 & 75.3 & & 2 & 75.3 \\
\hline & 3 & 76.5 & & 3 & 76.5 & & 3 & $77.9^{b)}$ & & 3 & $77.8^{b)}$ & & 3 & $77.8^{b)}$ \\
\hline & 4 & 79.3 & & 4 & 79.3 & & 4 & 71.3 & & 4 & 71.4 & & 4 & 71.5 \\
\hline & 5 & 76.9 & & 5 & 76.9 & & 5 & $78.0^{b)}$ & & 5 & $78.0^{b)}$ & & 5 & $78.0^{b)}$ \\
\hline & 6 & 61.9 & & 6 & 61.9 & & 6 & 62.6 & & 6 & 62.7 & & 6 & 62.7 \\
\hline \multirow[t]{6}{*}{ rham } & 1 & 102.9 & rham & 1 & 102.9 & & & & & & & & & \\
\hline & 2 & 72.2 & & 2 & 72.2 & & & & & & & & & \\
\hline & 3 & 72.4 & & 3 & 72.4 & & & & & & & & & \\
\hline & 4 & 73.8 & & 4 & 73.7 & & & & & & & & & \\
\hline & 5 & 70.7 & & 5 & 70.6 & & & & & & & & & \\
\hline & 6 & 17.9 & & 6 & 17.9 & & & & & & & & & \\
\hline \multicolumn{15}{|c|}{ Sugar on $\mathrm{C}-28^{a)}$} \\
\hline \multirow[t]{6}{*}{ glc } & 1 & 95.8 & glc & 1 & 95.8 & glc & 1 & 95.7 & glc & 1 & 95.7 & $\mathrm{glc}$ & 1 & 95.7 \\
\hline & 2 & 73.8 & & 2 & 73.8 & & 2 & 73.8 & & 2 & 73.8 & & 2 & 73.9 \\
\hline & 3 & 78.2 & & 3 & 78.2 & & 3 & $78.1^{b)}$ & & 3 & $78.2^{b}$ & & 3 & 78.2 \\
\hline & 4 & 70.9 & & 4 & 70.9 & & 4 & 70.9 & & 4 & 73.9 & & 4 & 71.1 \\
\hline & 5 & 78.1 & & 5 & 78.1 & & 5 & $78.1^{b)}$ & & 5 & $78.1^{b)}$ & & 5 & 78.6 \\
\hline & 6 & 69.4 & & 6 & 69.4 & & 6 & 69.5 & & 6 & 69.5 & & 6 & 62.9 \\
\hline \multirow[t]{6}{*}{ glc } & 1 & 104.3 & glc & 1 & 104.3 & glc & 1 & 104.6 & glc & 1 & 104.7 & & & \\
\hline & 2 & 75.3 & & 2 & 75.3 & & 2 & 75.1 & & 2 & 75.1 & & & \\
\hline & 3 & 76.8 & & 3 & 76.8 & & 3 & 79.7 & & 3 & 78.9 & & & \\
\hline & 4 & 79.5 & & 4 & 79.5 & & 4 & 71.5 & & 4 & 71.5 & & & \\
\hline & 5 & 76.7 & & 5 & 76.7 & & 5 & $78.0^{b)}$ & & 5 & $78.0^{b)}$ & & & \\
\hline & 6 & 61.9 & & 6 & 61.9 & & 6 & 62.7 & & 6 & 62.7 & & & \\
\hline \multirow[t]{6}{*}{ rham } & 1 & 102.9 & rham & 1 & 102.9 & & & & & & & & & \\
\hline & 2 & 72.2 & & 2 & 72.2 & & & & & & & & & \\
\hline & 3 & 72.4 & & 3 & 72.4 & & & & & & & & & \\
\hline & 4 & 73.7 & & 4 & 73.7 & & & & & & & & & \\
\hline & 5 & 70.6 & & 5 & 70.6 & & & & & & & & & \\
\hline & 6 & 17.9 & & 6 & 17.9 & & & & & & & & & \\
\hline
\end{tabular}

a) ara $=\alpha$-L-arabinopyranosyl; glc $=\beta$-D-glucopyranosyl; rham $=\alpha$-L-rhamnopyranosyl. $b$ ) May be reversed.

saponins. Thus, the structure of pastuchoside A (1) was established as $3 \beta-O-\{\alpha$-L-rhamnopyranosyl- $(1 \rightarrow 2)-[\alpha-\mathrm{L}-$ rhamnopyranosyl-( $1 \rightarrow 4)$ - $\beta$-D-glucopyranosyl- $(1 \rightarrow 4)]-\alpha$-Larabinopyranosyl $\}-28-O$ - $[\alpha$-L-rhamnopyranosyl- $(1 \rightarrow 4)-\beta$-Dglucopyranosyl- $(1 \rightarrow 6)-\beta$-D-glucopyranosyl]-hederagenin.

The molecular formula of pastuchoside $\mathrm{C}(\mathbf{5})$ was found to be $\mathrm{C}_{59} \mathrm{H}_{96} \mathrm{O}_{27}$ by ESI-HR-MS (quasi-molecular $[\mathrm{M}+\mathrm{Na}]^{+}$ ion $m / z$ 1259.5994; calculated $m / z$ for $\mathrm{C}_{59} \mathrm{H}_{96} \mathrm{NaO}_{27}$ : 1259.6037). Acid hydrolysis of 5 gave arabinose, glucose, rhamnose and hederagenin. ${ }^{13} \mathrm{C}-\mathrm{NMR}$ chemical shifts of $\mathrm{C}-3$ and $\mathrm{C}-28$ indicated that saponin was a bisdesmoside. Moreover, this spectrum showed five anomeric carbons at $\delta 106.1$, 105.2, 104.6, 101.9 and 95.7. Further analysis of the ${ }^{13} \mathrm{C}$ NMR data showed for sugar chains linked at C-3 similar chemical shifts with previously reported data of hederacolchiside $\mathrm{A}^{\prime}=$ hederacolchiside $\mathrm{A}_{1} \cdot{ }^{2)}$ It can be concluded that the structure of pastuchoside $\mathrm{C}$ was $3 \beta-O-\{\alpha-\mathrm{L}-$ rhamnopyranosyl-( $1 \rightarrow 2)$ - $[\beta$-D-glucopyranosyl- $(1 \rightarrow 4)]-\alpha$-L- arabinopyranosyl $\}-28-O$ - $[\beta$-D-glucopyranosyl-( $1 \rightarrow 6)-\beta$-Dglucopyranosyl]-hederagenin.

The molecular formula of pastuchoside D (7) was established as $\mathrm{C}_{59} \mathrm{H}_{96} \mathrm{O}_{26}$ by ESI-HR-MS $(\mathrm{m} / \mathrm{z} \quad 1243.6041$ $[\mathrm{M}+\mathrm{Na}]^{+}$, theoretical mass for $\left.\mathrm{C}_{59} \mathrm{H}_{96} \mathrm{NaO}_{26}, 1243.6088\right)$. Acid hydrolysis of 7 gave arabinose, glucose and rhamnose as sugars, and oleanolic acid as a genin. ${ }^{13} \mathrm{C}-\mathrm{NMR}$ spectrum data showed that 7 was a bisdesmoside having the same sugar chains linked at C-3 and C-28 with pastuchoside C. Thus, the structure of 7 was determined as $3 \beta-O-\{\alpha-\mathrm{L}-$ rhamnopyranosyl-( $1 \rightarrow 2)$ - $[\beta$-D-glucopyranosyl- $(1 \rightarrow 4)]-\alpha$-Larabinopyranosyl $\}-28-O$ - $[\beta$-D-glucopyranosyl- $(1 \rightarrow 6)-\beta$-Dglucopyranosyl]-oleanolate.

The ESI-HR-MS analysis of pastuchoside E (9) gave a quasi-molecular $[\mathrm{M}+\mathrm{Na}]^{+}$ion at $\mathrm{m} / z 1081.5569$ in agreement with a molecular formula of $\mathrm{C}_{53} \mathrm{H}_{86} \mathrm{O}_{21}\left(\mathrm{C}_{53} \mathrm{H}_{86} \mathrm{NaO}_{21}\right.$ requires 1081.5559 as theoretical mass). Acid hydrolysis of 9 yielded arabinose, glucose and rhamnose as sugars, and 
Table 4. ${ }^{13}$ C-NMR Data for Aglycone Moieties of Saponins 1, 3, 5, 7 and $9\left(\mathrm{CD}_{3} \mathrm{OD}\right)$

\begin{tabular}{|c|c|c|c|c|c|}
\hline $\mathrm{C}$ & 1 & 3 & 5 & 7 & 9 \\
\hline 1 & 39.8 & 40.0 & 39.8 & 40.0 & 40.0 \\
\hline 2 & 26.5 & 27.0 & 26.5 & 27.0 & 27.1 \\
\hline 3 & 82.3 & 90.6 & 82.3 & 90.5 & 90.6 \\
\hline 4 & 44.0 & 40.7 & 44.0 & 40.8 & 40.7 \\
\hline 5 & 48.2 & 57.2 & 48.3 & 57.2 & 57.2 \\
\hline 6 & 18.8 & 19.4 & 18.8 & 19.4 & 19.4 \\
\hline 7 & 33.2 & 33.3 & 33.2 & 33.2 & 33.2 \\
\hline 8 & 40.7 & 40.3 & 40.7 & 40.3 & 40.3 \\
\hline 9 & 49.2 & 49.0 & 49.2 & 49.1 & 49.1 \\
\hline 10 & 37.7 & 37.9 & 37.6 & 37.9 & 38.0 \\
\hline 11 & 24.6 & 24.6 & 24.6 & 24.6 & 24.6 \\
\hline 12 & 123.8 & 123.8 & 123.7 & 123.8 & 123.8 \\
\hline 13 & 144.9 & 144.9 & 144.9 & 144.9 & 144.9 \\
\hline 14 & 43.0 & 42.9 & 43.0 & 43.0 & 43.0 \\
\hline 15 & 28.9 & 28.9 & 28.9 & 28.9 & 28.9 \\
\hline 16 & 24.1 & 24.0 & 24.1 & 24.0 & 24.0 \\
\hline 17 & 48.1 & 48.1 & 48.1 & 48.1 & 48.1 \\
\hline 18 & 42.5 & 42.5 & 42.6 & 42.8 & 42.6 \\
\hline 19 & 47.2 & 47.2 & 47.2 & 47.3 & 47.3 \\
\hline 20 & 31.6 & 31.6 & 31.5 & 31.6 & 31.6 \\
\hline 21 & 34.9 & 34.9 & 34.9 & 34.9 & 34.9 \\
\hline 22 & 33.4 & 33.9 & 33.3 & 33.9 & 34.0 \\
\hline 23 & 65.1 & 28.6 & 65.0 & 28.7 & 28.7 \\
\hline 24 & 13.2 & 17.2 & 13.2 & 17.2 & 17.2 \\
\hline 25 & 16.6 & 16.2 & 16.6 & 16.2 & 16.2 \\
\hline 26 & 18.0 & 17.7 & 18.0 & 17.8 & 17.9 \\
\hline 27 & 26.4 & 26.3 & 26.4 & 26.3 & 26.4 \\
\hline 28 & 178.1 & 178.1 & 178.1 & 178.1 & 178.1 \\
\hline 29 & 33.5 & 33.5 & 33.5 & 33.5 & 33.6 \\
\hline 30 & 24.1 & 24.1 & 24.1 & 24.1 & 24.1 \\
\hline
\end{tabular}

oleanolic acid as a genin. The ${ }^{13} \mathrm{C}$ chemical shifts of $\mathrm{C}-3$ and $\mathrm{C}-28$ indicated that saponin 9 was a bisdesmoside. Moreover, ${ }^{13} \mathrm{C}$ shifts of sugars were indicative of similar sugar chains linked at C-3 with both saponins 5 and 7 . It can be concluded that the structure of pastuchoside $\mathrm{E}$ was $3 \beta-O-\{\alpha-\mathrm{L}-$ rhamnopyranosyl- $(1 \rightarrow 2)$-[ $\beta$-D-glucopyranosyl- $(1 \rightarrow 4)]-\alpha$-Larabinopyranosyl $\}-28-O-\beta$-D-glucopyranosyl-oleanolate.

\section{Experimental}

General Mass spectrometry analyses were performed on a Jeol JMS700 (Jeol LTD, Akishima, Tokyo, Japan) double focusing mass spectrometer, equipped with an electrospray ionization (ESI) source operating under positive ion mode. Samples diluted in $\mathrm{H}_{2} \mathrm{O} / \mathrm{CH}_{3} \mathrm{OH}(50 / 50)$ were introduced into the ESI interface via a syringe pump (PHD 2000 infusion, Harvard Apparatus, Holliston, MA, U.S.A.) at a $30 \mu 1 \cdot \mathrm{min}^{-1}$ flow rate. A $5-\mathrm{kV}$ acceleration voltage was applied and the elemental composition of ions was checked at a typical resolving power of 8000 (10\% valley) using a mixture of PEGs as internal standard. ${ }^{1} \mathrm{H}$ - and ${ }^{13} \mathrm{C}-\mathrm{NMR}$ spectra were recorded on a Bruker DRX-500 spectrometer in $\mathrm{CD}_{3} \mathrm{OD}$ solutions. TMS was used as an internal standard in ${ }^{1} \mathrm{H}$ and ${ }^{13} \mathrm{C}$ measurements. Standard Bruker pulse sequences were used for two-dimensional experiments (gradient selected COSY, HSQC, HSQC-TOCSY and HMBC). Microwave Pr KS-22E ( $850 \mathrm{~W}$, $2450 \mathrm{MHz}$ ) was used to dry the leaves. Melting points were determined on an Electrothermal IA 9300 apparatus. Optical rotations $[\alpha]_{\mathrm{D}}^{25}$ were measured on a Perkin-Elmer model 341 Orot polarimeter. TLC analyses of saponins and sugars were performed on precoated silica gel plates (Kiesegel 60F254, Merck) using the following solvent systems: $\mathrm{CHCl}_{3}-\mathrm{MeOH}-\mathrm{H}_{2} \mathrm{O}$ (26:14:3) [system 1]; $n$ - $\mathrm{BuOH}-\mathrm{HOAc}-\mathrm{H}_{2} \mathrm{O}(4: 1: 5)$ [system 2]; $\mathrm{CHCl}_{3}-$ $\mathrm{MeOH}(20: 1)$ [system 3]; $\mathrm{CH}_{2} \mathrm{Cl}_{2}-\mathrm{MeOH}-\mathrm{H}_{2} \mathrm{O}(50: 25: 5)$ [system 4]. Spots were detected by spraying the plates with phosphoric acid naphtoresorcinol for sugars and $\mathrm{H}_{2} \mathrm{SO}_{4}$ for saponins followed by heating at $110^{\circ} \mathrm{C}$.

Extraction and Separation Plant material was collected in the Lagodekhi region of Georgia (September 1999) and dried by microwave oven. A voucher specimen is kept in the Department of Pharmacobotany, Institute of Pharmacochemistry, Tbilisi, Georgia (leaves No. 97799). $500 \mathrm{~g}$ of crushed leaves was extracted with $80 \% \mathrm{MeOH}$ (21). After concentration, the aqueous layer was extracted by $n-\mathrm{BuOH}$, to obtain a crude extract of saponins $(100 \mathrm{~g})$, which was subjected to column chromatography on silica gel $\left(0.04-0.063 \mathrm{~mm}\right.$, Merck) and eluted with $\mathrm{CHCl}_{3}-\mathrm{MeOH}-\mathrm{H}_{2} \mathrm{O}$ (26:14:3) to afford 3 fractions. Fraction 3, containing the most polar triterpene saponins, was subjected repeatedly to low pressure liquid chromatography (ChromatoSPAC Prep 100, Lichroprep C-18, 15-25 $\mu \mathrm{m}$ ) and eluted with $\mathrm{MeOH}-\mathrm{H}_{2} \mathrm{O}(20 \%$ to $80 \%$ of $\mathrm{MeOH})$ to give $\mathbf{1}(15 \mathrm{mg}), \mathbf{2}(180 \mathrm{mg}), \mathbf{3}$ $(15 \mathrm{mg}), \mathbf{4}(150 \mathrm{mg}), 5$ (impure, $100 \mathrm{mg}), \mathbf{6}(110 \mathrm{mg}), \mathbf{7}(50 \mathrm{mg})$, mixture of 8 and $9(250 \mathrm{mg})$.

A fraction containing a mixture of $\mathbf{8}$ and $\mathbf{9}$ was purified by $\mathrm{CC}$ on silica gel $\left(0.04-0.063 \mathrm{~mm}\right.$, Merck) and eluted with $\mathrm{CHCl}_{3}-\mathrm{MeOH}-\mathrm{H}_{2} \mathrm{O}$ $(26: 14: 3)$ to yield $8(100 \mathrm{mg})$ and $9(50 \mathrm{mg})$.

The purification of $\mathbf{5}$ was carried out on polyamide column (SC 6 $0.07 \mathrm{~mm}$, Macherey-Nagel), with $50 \%$ of $\mathrm{MeOH}$ to obtain $50 \mathrm{mg}$ of 5 .

Acid Hydrolysis of 1, 3, 7 and 9 The saponin (4 mg) was heated with aqueous $10 \% \mathrm{HCl}(3 \mathrm{ml})$ in a sealed tube at $100^{\circ} \mathrm{C}$ for $4 \mathrm{~h}$. The sapogenin was extracted with $\mathrm{Et}_{2} \mathrm{O}$ and then the aqueous layer was neutralized with $\mathrm{N}, \mathrm{N}$-dioctylmethylamine $\left(10 \%\right.$ in $\left.\mathrm{CHCl}_{3}\right)$ and dried. The sapogenin and sugars were identified by TLC analyses with authentic samples in systems 3 and 4 , respectively.

Alkaline Hydrolysis of 1, 3, 7 and 9 The saponin (5 mg) in 5\% aqueous $\mathrm{KOH}(5 \mathrm{ml})$ was heated at $100^{\circ} \mathrm{C}$ in a sealed tube for $90 \mathrm{~min}$. After neutralization with $10 \% \mathrm{HCl}(\mathrm{pH} \mathrm{5})$ the prosapogenin was extracted with $n$-BuOH. TLC analyses were performed using systems 1 and 2 .

Pastuchoside A (1): White powder; $R f=0.09$ (in system 1). mp $198^{\circ} \mathrm{C}$; $[\alpha]_{\mathrm{D}}^{20}-16^{\circ}(c=0.1, \mathrm{MeOH})$. ESI-HR-MS $m / z: 1551.7191[\mathrm{M}+\mathrm{Na}]^{+}(\mathrm{Calcd}$ for $\mathrm{C}_{71} \mathrm{H}_{116} \mathrm{O}_{35}$ ). ${ }^{1} \mathrm{H}-\mathrm{NMR}$ data for sugar part, see Table $2 .{ }^{13} \mathrm{C}-\mathrm{NMR}$ data for sugar and aglycone parts see Tables 3 and 4 .

Pastuchoside B (3): White powder; $R f=0.13$ (in system 1). $\mathrm{mp} 212^{\circ} \mathrm{C}$; $[\alpha]_{\mathrm{D}}^{20}-40^{\circ}(c=0.1, \mathrm{MeOH})$. ESI-HR-MS $m / z: 1535.7280[\mathrm{M}+\mathrm{Na}]^{+}(\mathrm{Calcd}$ for $\left.\mathrm{C}_{71} \mathrm{H}_{116} \mathrm{O}_{34}\right) .{ }^{1} \mathrm{H}-\mathrm{NMR}$ data for sugar part, see Table $2 .{ }^{13} \mathrm{C}-\mathrm{NMR}$ data for sugar and aglycone parts see Tables 3 and 4 .

Pastuchoside C (5): White powder; $R f=0.12$ (in system 1). mp $201{ }^{\circ} \mathrm{C}$; $[\alpha]_{\mathrm{D}}^{20}-18^{\circ}(c=0.1, \mathrm{MeOH})$. ESI-HR-MS $m / z: 1259.5994[\mathrm{M}+\mathrm{Na}]^{+}(\mathrm{Calcd}$ for $\left.\mathrm{C}_{53} \mathrm{H}_{96} \mathrm{O}_{27}\right)$. ${ }^{1} \mathrm{H}-\mathrm{NMR}$ data for anomeric protons of sugar $\left(\mathrm{CD}_{3} \mathrm{OD}\right)$ $\delta: 5.34(\mathrm{~d}, J=7.9 \mathrm{~Hz}), 5.16(\mathrm{br} \mathrm{s}), 4.47(\mathrm{~d}, J=5.3 \mathrm{~Hz}), 4.43(\mathrm{~d}, J=7.6 \mathrm{~Hz})$, $4.32(\mathrm{~d}, J=7.8 \mathrm{~Hz}) \cdot{ }^{13} \mathrm{C}-\mathrm{NMR}$ data for sugar and aglycone parts see Tables 3 and 4.

Pastuchoside D (7): White powder; $R f=0.18$ (in system 1). $\mathrm{mp} 213^{\circ} \mathrm{C}$; $[\alpha]_{\mathrm{D}}^{20}-30^{\circ}(c=0.1, \mathrm{MeOH})$. ESI-HR-MS $m / z: 1243.6041[\mathrm{M}+\mathrm{Na}]^{+}(\mathrm{Calcd}$ for $\left.\mathrm{C}_{53} \mathrm{H}_{96} \mathrm{O}_{26}\right)$. ${ }^{1} \mathrm{H}-\mathrm{NMR}$ data for anomeric protons of sugar $\left(\mathrm{CD}_{3} \mathrm{OD}\right)$ $\delta: 5.34(\mathrm{~d}, J=7.9 \mathrm{~Hz}), 5.16$ (br s), $4.46(\mathrm{~d}, J=5.3 \mathrm{~Hz}), 4.43(\mathrm{~d}, J=7.6 \mathrm{~Hz})$, $4.33(\mathrm{~d}, J=7.8 \mathrm{~Hz}) .{ }^{13} \mathrm{C}-\mathrm{NMR}$ data for sugar and aglycone parts see Tables 3 and 4.

Pastuchoside E (9): White powder; $R f=0.36$ (in system 1). $\mathrm{mp} 205^{\circ} \mathrm{C}$; $[\alpha]_{\mathrm{D}}^{20}+25^{\circ}(c=0.1, \mathrm{MeOH})$. ESI-HR-MS $m / z: 1081.5569[\mathrm{M}+\mathrm{Na}]^{+}(\mathrm{Calcd}$ for $\left.\mathrm{C}_{53} \mathrm{H}_{86} \mathrm{O}_{21}\right)$. ${ }^{1} \mathrm{H}-\mathrm{NMR}$ data for anomeric protons of sugar $\left(\mathrm{CD}_{3} \mathrm{OD}\right)$ $\delta: 5.36(\mathrm{~d}, J=7.9 \mathrm{~Hz}), 5.16$ (br s), $4.48(\mathrm{~d}, J=5.3 \mathrm{~Hz}), 4.33(\mathrm{~d}, J=7.8 \mathrm{~Hz})$. ${ }^{13} \mathrm{C}-\mathrm{NMR}$ data for sugar and aglycone parts see Tables 3 and 4 .

Acknowledgements This work was supported by the INTAS program (project No. 97-0491). We wish thank Mr. Gilbert BOUDON for his technical assistance.

\section{References}

1) Sakartvelos Metsniereba, Tbilisi, 1984, IX, pp. 124-128.

2) Mshvildadze V. D., Dekanosidze G. E., Shashkof A. S., Kemertelidze E. P., Bioorganicheskaia Khimia, 19, 1001-1007 (1993).

3) Mshvildadze V. D., Elias R., Faure R., Debrauwer L., Dekanosidze G. E., Kemertelidze E. P., Balansard G., Chem. Pharm. Bull., 49, 752754 (2001)

4) Mshvildadze V. D., Favel A., Delmas F., Elias R., Faure R., Dekanosidze G. E., Kemertelidze E. P., Balansard G., Pharmazie, 55, $325-326$ (2000).

5) Delmas F., Di Giorgio C., Elias R., Gasquet M., Azas N., Mshvildadze V. D., Dekanosidze G. E., Kemertelidze E. P., Timon-David P., Planta Med., 66, 1-5 (2000).

6) Ridoux O., Di Giorgio C., Delmas F., Elias R., Mshvildadze V. D., Dekanosidze G. E., Kemertelidze E. P., Balansard G., Timon-David P., Phytother. Res., 15, 298-301 (2001).

7) Barthomeuf C., Debiton E., Mshvildadze V. D., Kemertelidze E. P., Balansard G., Planta Med., 68, 672-675 (2002).

8) Debiton E., Borel M., Communal Y., Mshvildadze V. D., Barthomeuf 
C., Melanoma Res., 14, 97-105 (2004).

9) Süleyman H., Mshvildadze V. D., Gepdiremen A., Elias R., Phytomedecine, 10, 370-374 (2003).

10) Dekanosidze G. E., Djikia O. D., Vulgalter M. M., Kemertelidze E. P., Khim. Prir. Soedi., 6, 747-749 (1984).

11) Dekanosidze G. E., Kemertelidze E. P., Khim. Prir. Soedi., 2, 259263 (1980).

12) Elias R., Diaz-Lanza A. M., Vidal-Ollivier E., Balansard G., Faure R., Babadjamian A., J. Nat. Prod., 54, 98-103 (1991).
13) Tschesche R., Schmidt W., Wulff Gr., Zhur. Naturforsch., 20, 708709 (1965).

14) Kay L. E., Kiefer P., Saarinen T., J. Am. Chem. Soc., 114, 1066310665 (1992).

15) John B. K., Plant D., Head S. L., Hurd R. E., J. Magn. Reson., 94, 664-669 (1991).

16) Wilker W., Leibfritz D., Kerssebaum R., Bermel W., Magn. Reson. Chem., 31, 287-292 (1993). 